ZU-TH 25/06

CERN-PH-TH/2006-271

IFT-23/2006

hep-ph/0612329

\title{
Four-Loop Anomalous Dimensions for Radiative Flavour-Changing Decays
}

\author{
Michał Czakon,1,2 Ulrich Haisch ${ }^{3}$ and Mikołaj Misiak ${ }^{4,5}$ \\ ${ }^{1}$ Institut für Theoretische Physik und Astrophysik, Universität Würzburg, \\ Am Hubland, D-97074 Würzburg, Germany. \\ ${ }^{2}$ Department of Field Theory and Particle Physics, Institute of Physics, \\ University of Silesia, Uniwersytecka 4, PL-40007 Katowice, Poland. \\ ${ }^{3}$ Institut für Theoretische Physik, Universität of Zürich, \\ Winterthurerstrasse 190, CH-8057 Zürich, Switzerland. \\ ${ }^{4}$ Theoretical Physics Division, CERN, CH-1211 Geneva 23, Switzerland. \\ ${ }^{5}$ Institute of Theoretical Physics, Warsaw University, \\ Hoża 69, PL-00681 Warsaw, Poland.
}

\begin{abstract}
We evaluate the complete four-loop anomalous dimension matrix that is necessary for determining the effective flavour-changing neutral current couplings $\bar{q} q^{\prime} \gamma$ and $\bar{q} q^{\prime} g$ at the nextto-next-to-leading order in QCD. The resulting $\mathcal{O}\left(\alpha_{s}^{2}\left(\mu_{b}\right)\right)$ correction to the $\bar{B} \rightarrow X_{s} \gamma$ branching ratio amounts to around $-2.9 \%$ for $\mu_{b}=5 \mathrm{GeV}$, and $-4.4 \%$ for $\mu_{b}=2.5 \mathrm{GeV}$.
\end{abstract}




\section{Introduction}

Flavour-Changing Neutral Current (FCNC) decays are well known to provide stringent constraints on extensions of the Standard Model (SM) because their SM amplitudes are loopsuppressed. An additional chirality suppression factor $m_{q} / M_{W}$ for radiative FCNC transitions $q \rightarrow q^{\prime} \gamma$ makes these processes particularly attractive. The chirality suppression may be offset in certain new-physics models like the Minimal Supersymmetric Standard Model with large $\tan \beta$ [1] or left-right models [2]. Precise SM calculations of the radiative flavour-changing decay rates are thus vital for our ability to derive constraints on new physics from these observables.

In the present paper, we evaluate the complete four-loop Anomalous Dimension Matrix (ADM) that is necessary for calculating the effective FCNC couplings $\bar{q} q^{\prime} \gamma$ and $\bar{q} q^{\prime} g$ at the Next-to-Next-to-Leading Order (NNLO) in QCD. Our main motivation is contributing to the NNLO calculation of the inclusive $\bar{B} \rightarrow X_{s} \gamma$ branching ratio that is generated at the quark level by the $b \rightarrow s \gamma$ transition. For this decay, both the current experimental errors and the non-perturbative effects are smaller than the perturbative NNLO corrections [3]. Our four-loop $\mathcal{O}\left(\alpha_{s}^{2}\left(\mu_{b}\right)\right)$ contributions turn out to suppress $\mathcal{B}\left(\bar{B} \rightarrow X_{s} \gamma\right)$ by around $2.9 \%$ for $\mu_{b}=5 \mathrm{GeV}$, and $4.4 \%$ for $\mu_{b}=2.5 \mathrm{GeV}$. The dominant part of this effect has already been included in the phenomenological NNLO analysis of Refs. [3,4].

The article is organized as follows. In Section 2, we introduce the relevant effective Lagrangian and describe the structure of the Renormalization Group Equations (RGEs) for the Wilson coefficients. In Section 3, the bare four-loop calculation is described. Section 4 is devoted to the calculation of subdivergences. Our final results for the renormalization constants and ADMs are given in Section 5. The numerical size of the four-loop effects is analyzed in Section 6. We summarize in Section 7. Appendix A contains solutions to the RGEs for all the Wilson coefficients that matter for $b \rightarrow s \gamma$ at the NNLO before including higher-order electroweak corrections.

\section{Effective Lagrangian}

All the FCNC processes that have been observed so far are generated at the electroweak scale $\mu_{0} \sim M_{W}, m_{t}$, while the relevant momentum transfer scale $\mu_{f}$ is much lower. Large logarithm

$\left(\alpha_{s} \ln \mu_{0}^{2} / \mu_{f}^{2}\right)^{n}$ resummation at each order of the perturbation series in $\alpha_{s}\left(\mu_{f}\right)$ is necessary to obtain viable results. It is achieved by making use of the RGEs in an effective theory that arises from the SM after decoupling of the heavy electroweak bosons and the top quark [5].

The effective Lagrangian that matters for the $q \rightarrow q^{\prime} \gamma$ and $q \rightarrow q^{\prime} g$ transitions at the leading order in the electroweak interactions is given below. For definiteness, we specify the quark flavours as in the $b \rightarrow s \gamma$ case.

$$
\mathcal{L}_{\text {eff }}=\mathcal{L}_{\mathrm{QCD} \times \mathrm{QED}}(u, d, s, c, b)+\frac{4 G_{F}}{\sqrt{2}} V_{t s}^{*} V_{t b} \sum_{i=1}^{8} C_{i}(\mu) Q_{i}+\ldots
$$

Here, $G_{F}$ and $V_{i j}$ stand for the Fermi coupling constant and the quark mixing matrix elements, respectively. The Wilson coefficients $C_{i}(\mu)$ play the role of coupling constants at the effective 
weak vertices (operators) $Q_{i}$. The operator basis is chosen as in Ref. [6] to avoid difficulties with $\gamma_{5}$ in dimensional regularization.

$$
\begin{aligned}
Q_{1} & =\left(\bar{s} \gamma_{\mu} T^{a} P_{L} c\right)\left(\bar{c} \gamma^{\mu} T^{a} P_{L} b\right) \\
Q_{2} & =\left(\bar{s} \gamma_{\mu} P_{L} c\right)\left(\bar{c} \gamma^{\mu} P_{L} b\right) \\
Q_{3} & =\left(\bar{s} \gamma_{\mu} P_{L} b\right) \sum_{q}\left(\bar{q} \gamma^{\mu} q\right) \\
Q_{4} & =\left(\bar{s} \gamma_{\mu} T^{a} P_{L} b\right) \sum_{q}\left(\bar{q} \gamma^{\mu} T^{a} q\right) \\
Q_{5} & =\left(\bar{s} \gamma_{\mu_{1}} \gamma_{\mu_{2}} \gamma_{\mu_{3}} P_{L} b\right) \sum_{q}\left(\bar{q} \gamma^{\mu_{1}} \gamma^{\mu_{2}} \gamma^{\mu_{3}} q\right), \\
Q_{6} & =\left(\bar{s} \gamma_{\mu_{1}} \gamma_{\mu_{2}} \gamma_{\mu_{3}} T^{a} P_{L} b\right) \sum_{q}\left(\bar{q} \gamma^{\mu_{1}} \gamma^{\mu_{2}} \gamma^{\mu_{3}} T^{a} q\right), \\
Q_{7} & =\frac{e}{16 \pi^{2}}\left[\bar{s} \sigma^{\mu \nu}\left(m_{s} P_{L}+m_{b} P_{R}\right) b\right] F_{\mu \nu}, \\
Q_{8} & =\frac{g}{16 \pi^{2}}\left[\bar{s} \sigma^{\mu \nu}\left(m_{s} P_{L}+m_{b} P_{R}\right) T^{a} b\right] G_{\mu \nu}^{a},
\end{aligned}
$$

where $P_{L, R}=\left(1 \mp \gamma_{5}\right) / 2$. For simplicity, terms proportional to the small $V_{u b}$ mixing have been neglected here. The dots on the r.h.s. of Eq. (2.1) stand for ultraviolet (UV) counterterms. Apart from $Q_{1}, \ldots, Q_{8}$, the counterterms contain unphysical operators that matter only off-shell and/or in $D \neq 4$ spacetime dimensions (see Section 4).

Instead of the original $C_{i}(\mu)$, it is more convenient to work with certain linear combinations of them, the so-called "effective" Wilson coefficients

$$
C_{i}^{\mathrm{eff}}(\mu)= \begin{cases}C_{i}(\mu), & \text { for } i=1, \ldots, 6, \\ C_{7}(\mu)+\sum_{j=1}^{6} y_{j} C_{j}(\mu), & \text { for } i=7, \\ C_{8}(\mu)+\sum_{j=1}^{6} z_{j} C_{j}(\mu), & \text { for } i=8 .\end{cases}
$$

The numbers $y_{j}$ and $z_{j}$ are defined so that the $b \rightarrow s \gamma$ and $b \rightarrow s g$ amplitudes at the Leading Order (LO) in QCD are proportional to $C_{7}^{\text {eff }}$ and $C_{8}^{\text {eff }}$, respectively [7]. In the $\overline{\mathrm{MS}}$ scheme with fully anticommuting $\gamma_{5}$ which is used in our calculation, one finds, $\vec{y}=\left(0,0,-\frac{1}{3},-\frac{4}{9},-\frac{20}{3},-\frac{80}{9}\right)$ and $\vec{z}=\left(0,0,1,-\frac{1}{6}, 20,-\frac{10}{3}\right)[6]$.

The RGEs for $C_{i}^{\text {eff }}(\mu)$

$$
\mu \frac{d}{d \mu} C_{i}^{\mathrm{eff}}(\mu)=C_{j}^{\mathrm{eff}}(\mu) \gamma_{j i}^{\mathrm{eff}}(\mu)
$$

are governed by the ADM

$$
\hat{\gamma}^{\mathrm{eff}}(\mu)=\sum_{n \geq 0} \widetilde{\alpha}^{n+1} \hat{\gamma}^{(n) \mathrm{eff}}
$$

where $\widetilde{\alpha}=\alpha_{s}(\mu) /(4 \pi)$. This matrix is determined from the effective theory renormalization constants. The matrices $\hat{\gamma}^{(n) \text { eff }}$ have a block-triangular structure

$$
\hat{\gamma}^{(n) \text { eff }}=\left(\begin{array}{cc}
A_{6 \times 6}^{(n)} & B_{6 \times 2}^{(n)} \\
0_{2 \times 6} & C_{2 \times 2}^{(n)}
\end{array}\right) .
$$

The down-left block vanishes because the dipole operators $Q_{7}$ and $Q_{8}$ are actually dimensionfive ones, and thus generate no UV divergences in dimension-six four-quark amplitudes. The 




Figure 1: One of the $\mathcal{O}\left(10^{4}\right)$ calculated four-loop diagrams.

diagonal blocks $A^{(n)}$ and $C^{(n)}$ are found from $(n+1)$-loop renormalization constants. Nonvanishing contributions to the off-diagonal blocks $B^{(n)}$ arise at two and more loops only. Once the normalization conventions for $Q_{7}$ and $Q_{8}$ are chosen as in Eq. (2.2), the blocks $B^{(n)}$ contain information on $(n+2)$-loop renormalization 1

The complete matrices $\hat{\gamma}^{(0) \text { eff }}$ and $\hat{\gamma}^{(1) \text { eff }}$ together with the relevant references can be found in Ref. [6]. We quote these results in Section 5. The three-loop block $B^{(1)}$ was confirmed in Ref. [8]. At the NNLO, one needs to know the full $8 \times 8$ matrix $\hat{\gamma}^{(2) e f f}$. The three-loop blocks $A^{(2)}$ and $C^{(2)}$ were calculated in Refs. [9] and [10], respectively. In the present paper, we evaluate the four-loop block $B^{(2)}$.

\section{Bare Four-Loop Calculation}

The renormalization constants contributing to the matrix $B^{(2)}$ are found after subloop renormalization from the UV-divergent parts of four-loop diagrams like the one shown in Fig. 1. Each of the operators $Q_{1}, \ldots Q_{6}$ must be considered in the effective four-quark vertex, and the external gauge boson can be either a photon or a gluon. The overall number of relevant four-loop diagrams turns out to be 21986, when different color and gamma matrix structures are treated together.

In order to simplify the calculation as much as possible, an infrared (IR) rearrangement as in Refs. [11-14] has been applied. In this approach, a Taylor expansion in the external momenta is performed after introducing a common mass in all the propagator denominators. The order of the expansion is determined by the dimensions of the operator insertion and the Green's function. In the present case, up to two derivatives have to be applied. Afterwards, the problem is reduced to evaluation of single-scale fully massive vacuum integrals.

\footnotetext{
1 Inverse powers of coupling constants are sometimes used in the dipole operator normalization to make $\hat{\gamma}^{(n) \text { eff }}$ a purely $(n+1)$-loop object. Such a choice is convenient at intermediate steps (see Section 5), but the final results for the RGEs are more compact when the normalization as in Eq. (2.2) is applied.
} 
In principle, the contribution of any graph can be mapped onto the following Dirac structures

$$
\begin{aligned}
S_{j}=\left(\gamma_{\mu} \not p k, \gamma_{\mu}(p \cdot k), \gamma_{\mu} p^{2}, \gamma_{\mu} k^{2}, \not p k_{\mu}, \not p p_{\mu}, \not k p_{\mu}, \not k k_{\mu},\right. \\
\left.m_{b} \not k \gamma_{\mu}, m_{b} \gamma_{\mu} \not k, m_{b} p \gamma_{\mu}, m_{b} \gamma_{\mu} \not p, M^{2} \gamma_{\mu}\right)_{j},
\end{aligned}
$$

where $p$ and $k$ stand for the incoming $b$-quark and the outgoing photon/gluon momenta, respectively, whereas $M$ denotes the regulator mass. However, due to the huge computational resource requirements, only the coefficients at the structures $S_{j}$ for $j=7,9,10,11$ have been calculated. Only these four structures are needed to determine the sought ADM $B^{(2)}$ (see Section 4).

Comparing the vertex diagram structure and the Taylor expansion depth with those needed in the evaluation of the four-loop $\beta$-function with a single gauge parameter, we note that the maximal powers of numerators and denominators in the integrals are the same. This has allowed us to use the solution of the integration-by-parts identities and the master integrals determined in Ref. [14]. All the salient details of the employed techniques can be found in that paper.

\section{Subdivergences}

Considering renormalization of all the operators up to dimension six that can arise in the effective theory (2.1) is necessary for proper subtraction of subdivergences in our calculation. Since the four-loop diagrams are evaluated in the usual Feynman-'t Hooft gauge (without background fields), in $D=4-2 \epsilon$ dimensions and off-shell, the following types of counterterms must be taken into account apart from the usual QCD ones:

- the original ("physical") operators from Eq. (2.2),

- gauge-invariant operators that vanish by the $\mathrm{QCD} \times \mathrm{QED}$ Equations Of Motion (EOM),

- gauge-variant EOM-vanishing operators,

- BRST-exact operators, i.e. operators that can be written as Becchi-Rouet-Stora-Tyutin variations of other operators, and

- evanescent operators, i.e. operators that vanish in $D=4$ spacetime dimensions by Diracalgebra identities, but have to be included for $D \neq 4$.

One begins with writing down all the possible operators of dimension up to six with proper flavour quantum numbers that fall into the above five classes. The potentially infinite set of evanescent objects is reduced to a finite one at each given order of the perturbation series once the prescription of Ref. [15] is applied.

The long list of relevant operators can be further reduced by taking into account that the SM Lagrangian is invariant under the following discrete transformations of its fields and parameters (already after the electroweak symmetry breaking and mass matrix diagonalization):

$$
\text { - } b \leftrightarrow s, m_{b} \leftrightarrow m_{s}, V_{Q b} \leftrightarrow V_{Q s}(Q=u, c, t) .
$$


- $C P$ transformation and $V \rightarrow V^{*}$.

A combination of these two transformations maps the $\Delta B=-\Delta S=1$ interaction terms onto themselves, so it must leave $\mathcal{L}_{\text {eff }}(2.1)$ invariant. The relative plus sign between the $m_{s}$ and $m_{b}$ terms in the dipole operators $Q_{7}$ and $Q_{8}$ is fixed by this very symmetry. Once the operators are determined, terms proportional to $m_{s}$ are often neglected. Their effect on the $\bar{B} \rightarrow X_{s} \gamma$ branching ratio is negligible $\left(\mathcal{O}\left(m_{s}^{2} / m_{b}^{2}\right)\right)$ but they matter for the CP asymmetry. Some details on selecting the right number of gauge-invariant operators have been given in Section 5.3 of Ref. [16].

Explicit expressions for all the operators that satisfy the above-mentioned conditions can be found in Refs. [8,9], so we do not repeat them here. Let us only note that Section 2 of Ref. [8] contains all the EOM-vanishing operators, including the ones suppressed by the QED coupling $e$, and the one that gets generated at three loops only $\left(Q_{22}\right)$. On the other hand, the only possible BRST-exact operator and all the evanescent operators that we need here are given in Section 3 of Ref. [9]. The BRST-exact operator remains irrelevant. In particular, we have checked that the three-loop analogue of the two-loop amplitude described in Fig. 3 of Ref. [9] turns out to mysteriously vanish, as well.

Apart from all the "real" off-shell counterterms that have been mentioned so far, we encounter certain spurious ones that arise due to our particular IR rearrangement. The dimensionthree gluon-mass-like counterterm was discussed in detail in Refs. [11,12]. Spurious dimensionfour flavour-changing operators that are proportional to the IR-regulator mass (see Eq. (18) of Ref. [8]) are included, too.

The completeness of the full operators basis has been verified in many ways. For instance, since the number of operators contributing at the tree level to the $b \rightarrow s \gamma$ transition is smaller than the number of independent Dirac structures $S_{j}$ (3.1), there exist seven linear relations between coefficients at these structures in the renormalized amplitude. All these relations together with three analogous ones for $b \rightarrow s g$ have been successfully tested up to three loops [6, $8,9,17]$. In the present calculation, we determine coefficients at four of the structures $S_{j}$ only, which leaves us with just one linear relation for $b \rightarrow s \gamma$ and none for $b \rightarrow s g$. Therefore, we essentially rely on the fact that the operator basis is indeed complete.

Once the $(n \leq 3)$-loop diagrams with counterterms are added to the bare four-loop ones, we obtain an expression of the form

$$
A_{7} \not k p_{\mu}+m_{b}\left(A_{9} \not k \gamma_{\mu}+A_{10} \gamma_{\mu} \not k+A_{11} p \gamma_{\mu}\right)
$$

where the coefficients $A_{i}$ contain UV-poles only. These poles need to be canceled by tree-level counterterms. First, we subtract such tree-level counterterms that originate from products of the usual QCD renormalization constants with the $(n \leq 3)$-loop operator ones, which modifies the coefficients in Eq. (4.1). Let us call the new coefficients $A_{i}^{\prime}$. The linear combination

$$
\frac{1}{4} A_{7}^{\prime}-\frac{1}{2} A_{9}^{\prime}+\frac{1}{2} A_{10}^{\prime}-\frac{1}{2} A_{11}^{\prime}
$$

gives us the sought four-loop contributions to the renormalization constants $Z_{i 7}$ and $Z_{i 8}$ (for $i=1, \ldots 6)$ in the photonic and gluonic cases, respectively. The single consistency relation that 
is successfully tested in the photonic case reads $A_{9}^{\prime}+A_{10}^{\prime}+A_{11}^{\prime}=0$. Proving that the projection (4.2) is the right one requires studying Feynman rules for each operator in the full basis and verifying that only $Q_{7}$ or $Q_{8}$ can contribute to this particular combination at the tree level.

A very powerful test of the results in Eq. (4.1) is provided by the so-called locality constraints. Let us denote the $n$-loop $1 / \epsilon^{m}$-contribution to $A_{i}$ by $A_{i}^{(n m)}$, i.e.

$$
A_{i}=\sum_{n, m=1}^{4} \frac{\mu^{2 n \epsilon}}{\epsilon^{m}} A_{i}^{(n m)}
$$

The quantities $A_{i}^{(n m)}$ must satisfy many relations to ensure that all the $\left(\ln ^{k} \mu\right) / \epsilon^{j}$ poles cancel out in $A_{i}$. Such a cancellation is necessary for the counterterms to remain polynomial in external momenta, i.e. local in the position space. The explicit relations read

$$
\begin{aligned}
12 A_{i}^{(44)} & =-3 A_{i}^{(14)}=2 A_{i}^{(24)}=-3 A_{i}^{(34)} \\
6 A_{i}^{(43)} & =-A_{i}^{(23)}-3 A_{i}^{(33)} \\
8 A_{i}^{(43)} & =A_{i}^{(13)}-3 A_{i}^{(33)} \\
4 A_{i}^{(42)} & =-A_{i}^{(12)}-2 A_{i}^{(22)}-3 A_{i}^{(32)} .
\end{aligned}
$$

All these relations have been checked to hold in the photonic and gluonic cases for each of the six operator insertions. One should realize that testing the $1 / \epsilon^{2}$ poles in a four-loop amplitude constitutes a much more sensitive cross-check of the calculation than doing the same in a twoloop amplitude. Since $A_{i}^{4 m}$ and $A_{i}^{1 m}$ have been evaluated with the help of different routines written by different authors, we can be practically sure that our calculation is free of errors like missing a diagram or taking it with a wrong overall factor.

\section{Renormalization Constants and ADMs}

The counterterms in the effective Lagrangian that we have calculated can be written in the following form

$$
\Delta \mathcal{L}_{\text {eff }}^{\text {count }}=\frac{4 G_{F}}{\sqrt{2}} V_{t s}^{*} V_{t b} \sum_{i=1}^{6} C_{i}\left(Z_{i 7} X_{7}+Z_{i 8} X_{8}\right),
$$

where

$$
X_{7}=\frac{16 \pi^{2}}{\left(Z_{g} g\right)^{2}} Z_{0} Q_{7} \quad \text { and } \quad X_{8}=\frac{16 \pi^{2}}{\left(Z_{g} g\right)^{2}} Z_{0} Z_{g} Z_{G}^{1 / 2} Q_{8} .
$$

Here, $Z_{0}, Z_{g}$ and $Z_{G}$ are the usual QCD renormalization constants of the mass term, gauge coupling and the gluon field

$$
(m \bar{\psi} \psi)_{B}=Z_{0} m \bar{\psi} \psi, \quad g_{B}=Z_{g} g, \quad G_{B}^{a \mu}=Z_{G}^{1 / 2} G^{a \mu} .
$$

Below, we give our results for $Z_{i 7}$ and $Z_{i 8}$ up to four loops. The "trivial" terms proportional to $\ln (4 \pi)$ or to the Euler constant $\gamma_{E}$ are omitted, as they have no effect on the $\overline{\mathrm{MS}}$ anomalous 
dimensions. The Riemann zeta function value $\zeta(3) \simeq 1.20206$ is denoted by $\zeta_{3}$. The number of active flavours in the effective theory and the sum of their charges are denoted by $f$ and $\bar{Q}$, respectively. For $Z_{17}$ and $Z_{27}$, the charge $Q_{u}$ of the charm quark is retained arbitrary. The incoming quark charge is set to $-\frac{1}{3}$ in all the expressions for $Z_{i 7}$, which means that they correspond to the down-type quark $\bar{q} q^{\prime} \gamma$ coupling after substituting $Q_{u}=\frac{2}{3}$. For the external up-type quark case, one should multiply all the terms in $Z_{i 7}$ that come with no charge factor by -2 , and then replace $Q_{u}$ by $Q_{d}=-\frac{1}{3}$. The same rule applies to the matrices $B^{(n)}$ that are given in Eqs. (5.9) -(5.11) at the end of this section. We find

$$
\begin{aligned}
& Z_{17}=-\frac{\widetilde{\alpha}^{2}}{\epsilon}\left(\frac{4}{243}+\frac{1}{3} Q_{u}\right)+\frac{\widetilde{\alpha}^{3}}{\epsilon}\left\{\frac{1}{\epsilon}\left[-\frac{274}{6561}-\frac{40}{2187} f+\left(\frac{130}{27}-\frac{8}{27} f\right) Q_{u}\right]+\frac{3239}{6561}-\frac{32}{6561} f-\left(\frac{352}{81}\right.\right. \\
& \left.\left.-\frac{1}{81} f\right) Q_{u}\right\}+\frac{\widetilde{\alpha}^{4}}{\epsilon}\left\{\frac{1}{\epsilon^{2}}\left[\frac{97508}{59049}+\frac{487}{6561} f-\frac{104}{6561} f^{2}-\left(\frac{1556}{27}-\frac{193}{27} f+\frac{2}{9} f^{2}\right) Q_{u}-\frac{5}{108} \bar{Q}\right]\right. \\
& +\frac{1}{\epsilon}\left[-\frac{93353}{19683}+\frac{94513}{236196} f-\frac{100}{19683} f^{2}+\left(\frac{47807}{648}-\frac{599}{81} f+\frac{1}{81} f^{2}\right) Q_{u}-\frac{245}{972} \bar{Q}\right]-\frac{1385935}{8503056}-\frac{20435}{6561} \zeta_{3} \\
& +\left(\frac{46625}{354294}+\frac{91}{729} \zeta_{3}\right) f+\frac{70}{19683} f^{2}+\left[\frac{14797}{432}+\frac{8381}{162} \zeta_{3}+\left(\frac{11969}{5832}-\frac{8}{81} \zeta_{3}\right) f+\frac{79}{1458} f^{2}\right] Q_{u} \\
& \left.+\left(\frac{3695}{3888}-\frac{25}{54} \zeta_{3}\right) \bar{Q}\right\} \text {, } \\
& Z_{18}=\frac{167}{648} \frac{\widetilde{\alpha}^{2}}{\epsilon}+\frac{\widetilde{\alpha}^{3}}{\epsilon}\left[\frac{1}{\epsilon}\left(-\frac{6098}{2187}+\frac{1373}{5832} f\right)-\frac{40655}{34992}+\frac{431}{34992} f\right]+\frac{\widetilde{\alpha}^{4}}{\epsilon}\left[\frac{1}{\epsilon^{2}}\left(\frac{11512021}{314928}-\frac{313309}{52488} f+\frac{839}{3888} f^{2}\right)\right. \\
& \left.+\frac{1}{\epsilon}\left(\frac{7084465}{839808}+\frac{575321}{314928} f+\frac{659}{34992} f^{2}\right)-\frac{490821325}{22674816}-\frac{368107}{8748} \zeta_{3}-\left(\frac{2647291}{944784}+\frac{10381}{3888} \zeta_{3}\right) f-\frac{1955}{52488} f^{2}\right] \text {, } \\
& Z_{27}=\frac{\widetilde{\alpha}^{2}}{\epsilon}\left(\frac{8}{81}+2 Q_{u}\right)+\frac{\widetilde{\alpha}^{3}}{\epsilon}\left\{\frac{1}{\epsilon}\left[-\frac{4636}{2187}+\frac{80}{729} f-\left(\frac{206}{9}-\frac{16}{9} f\right) Q_{u}\right]+\frac{6698}{2187}+\frac{64}{2187} f+\left(\frac{128}{27}\right.\right. \\
& \left.\left.-\frac{2}{27} f\right) Q_{u}\right\}+\frac{\widetilde{\alpha}^{4}}{\epsilon}\left\{\frac{1}{\epsilon^{2}}\left[\frac{560390}{19683}-\frac{7400}{2187} f+\frac{208}{2187} f^{2}+\left(\frac{4379}{18}-\frac{323}{9} f+\frac{4}{3} f^{2}\right) Q_{u}+\frac{5}{18} \bar{Q}\right]\right. \\
& +\frac{1}{\epsilon}\left[-\frac{735973}{13122}+\frac{173111}{39366} f+\frac{200}{6561} f^{2}-\left(\frac{39427}{216}-\frac{613}{27} f+\frac{2}{27} f^{2}\right) Q_{u}+\frac{245}{162} \bar{Q}\right]+\frac{3142663}{1417176}+\frac{5068}{2187} \zeta_{3} \\
& -\left(\frac{79754}{59049}+\frac{146}{243} \zeta_{3}\right) f-\frac{140}{6561} f^{2}+\left[\frac{11699}{36}-\frac{6140}{27} \zeta_{3}-\left(\frac{17153}{972}+\frac{128}{27} \zeta_{3}\right) f-\frac{79}{243} f^{2}\right] Q_{u} \\
& \left.+\left(-\frac{3695}{648}+\frac{25}{9} \zeta_{3}\right) \bar{Q}\right\} \text {, } \\
& Z_{28}=\frac{19}{27} \frac{\widetilde{\alpha}^{2}}{\epsilon}+\frac{\widetilde{\alpha}^{3}}{\epsilon}\left[\frac{1}{\epsilon}\left(-\frac{23063}{2916}+\frac{571}{972} f\right)+\frac{5167}{2916}-\frac{917}{5832} f\right]+\frac{\widetilde{\alpha}^{4}}{\epsilon}\left[\frac{1}{\epsilon^{2}}\left(\frac{10632025}{104976}-\frac{63787}{4374} f+\frac{161}{324} f^{2}\right)\right. \\
& \left.-\frac{1}{\epsilon}\left(\frac{11481667}{139968}-\frac{714847}{52488} f+\frac{1307}{5832} f^{2}\right)+\frac{252836197}{3779136}-\frac{487691}{5832} \zeta_{3}-\left(\frac{6112793}{629856}+\frac{1679}{648} \zeta_{3}\right) f-\frac{2489}{17496} f^{2}\right] \text {, } \\
& Z_{37}=\frac{16}{81} \frac{\widetilde{\alpha}^{2}}{\epsilon}+\frac{\widetilde{\alpha}^{3}}{\epsilon}\left[\frac{1}{\epsilon}\left(-\frac{18236}{2187}+\frac{160}{729} f+4 \bar{Q}\right)+\frac{49144}{2187}-\frac{1816}{2187} f-\frac{56}{9} \bar{Q}\right]+\frac{\widetilde{\alpha}^{4}}{\epsilon}\left\{\frac { 1 } { \epsilon ^ { 2 } } \left[\frac{2436463}{19683}-\frac{27850}{2187} f\right.\right. \\
& \left.+\frac{416}{2187} f^{2}-\left(\frac{1265}{18}-\frac{14}{3} f\right) \bar{Q}\right]+\frac{1}{\epsilon}\left[-\frac{10574227}{26244}+\frac{1737559}{39366} f-\frac{5864}{6561} f^{2}+\left(\frac{76669}{648}-\frac{22}{3} f\right) \bar{Q}\right] \\
& \left.+\quad \frac{141396541}{708588}-\frac{59074}{2187} \zeta_{3}-\left(\frac{13789403}{472392}+\frac{598}{243} \zeta_{3}\right) f-\frac{244}{6561} f^{2}+\left[\frac{18007}{324}-\frac{56}{3} \zeta_{3}-\left(\frac{1}{27}+\frac{8}{9} \zeta_{3}\right) f\right] \bar{Q}\right\}, \\
& Z_{38}=\frac{92}{27} \frac{\widetilde{\alpha}^{2}}{\epsilon}+\frac{\widetilde{\alpha}^{3}}{\epsilon}\left[\frac{1}{\epsilon}\left(-\frac{63293}{1458}+\frac{4085}{972} f\right)+\frac{87031}{1458}-\frac{3361}{1458} f\right]+\frac{\widetilde{\alpha}^{4}}{\epsilon}\left[\frac{1}{\epsilon^{2}}\left(\frac{32831857}{52488}-\frac{3821329}{34992} f+\frac{2891}{648} f^{2}\right)\right. \\
& +\frac{1}{\epsilon}\left(-\frac{97744615}{69984}+\frac{73524781}{419904} f-\frac{19453}{5832} f^{2}\right)+\frac{176999365}{118098}-\frac{1095749}{2916} \zeta_{3}-\left(\frac{248941103}{5038848}+\frac{505}{162} \zeta_{3}\right) f \\
& \left.+\left(\frac{27313}{34992}-\frac{5}{18} \zeta_{3}\right) f^{2}\right] \text {, } \\
& Z_{47}=-\frac{\widetilde{\alpha}^{2}}{\epsilon}\left(\frac{170}{243}-\frac{8}{81} f\right)+\frac{\widetilde{\alpha}^{3}}{\epsilon}\left[\frac{1}{\epsilon}\left(\frac{59824}{6561}-\frac{148}{81} f+\frac{80}{729} f^{2}+\frac{5}{3} \bar{Q}\right)-\frac{104951}{6561}+\frac{2774}{6561} f+\frac{16}{2187} f^{2}-\frac{70}{27} \bar{Q}\right]
\end{aligned}
$$




$$
\begin{aligned}
& +\frac{\widetilde{\alpha}^{4}}{\epsilon}\left\{\frac{1}{\epsilon^{2}}\left[-\frac{6446822}{59049}+\frac{521993}{19683} f-\frac{17827}{6561} f^{2}+\frac{208}{2187} f^{3}-\left(\frac{850}{27}-\frac{20}{9} f\right) \bar{Q}\right]+\frac{1}{\epsilon}\left[\frac{12186259}{39366}-\frac{7760143}{236196} f\right.\right. \\
& \left.+\quad \frac{58249}{39366} f^{2}+\frac{56}{6561} f^{3}+\left(\frac{206399}{3888}-\frac{1175}{324} f\right) \bar{Q}\right]-\frac{895573151}{2125764}-\frac{439534}{6561} \zeta_{3}+\left(\frac{21721465}{708588}-\frac{8165}{2187} \zeta_{3}\right) f \\
& \left.-\left(\frac{71315}{59049}+\frac{160}{243} \zeta_{3}\right) f^{2}-\frac{148}{6561} f^{3}-\left[\frac{42823}{7776}-\frac{1355}{108} \zeta_{3}-\left(\frac{305}{216}-\frac{25}{27} \zeta_{3}\right) f\right] \bar{Q}\right\}, \\
& Z_{48}=-\frac{\widetilde{\alpha}^{2}}{\epsilon}\left(\frac{427}{324}+\frac{37}{216} f\right)+\frac{\widetilde{\alpha}^{3}}{\epsilon}\left[\frac{1}{\epsilon}\left(\frac{84661}{4374}+\frac{71}{36} f-\frac{185}{972} f^{2}\right)-\frac{540209}{17496}-\frac{40091}{34992} f-\frac{253}{2916} f^{2}\right] \\
& +\frac{\widetilde{\alpha}^{4}}{\epsilon}\left[\frac{1}{\epsilon^{2}}\left(-\frac{44723201}{157464}-\frac{590221}{34992} f+\frac{216085}{34992} f^{2}-\frac{259}{1296} f^{3}\right)+\frac{1}{\epsilon}\left(\frac{238543801}{419904}-\frac{4684067}{314928} f-\frac{1281229}{419904} f^{2}\right.\right. \\
& \left.\left.-\frac{253}{1944} f^{3}\right)-\frac{5633994047}{22674816}+\frac{910901}{4374} \zeta_{3}+\left(\frac{566791855}{30233088}+\frac{208123}{5832} \zeta_{3}\right) f+\left(\frac{2176469}{1259712}+\frac{115}{81} \zeta_{3}\right) f^{2}+\frac{505}{34992} f^{3}\right], \\
& Z_{57}=-\frac{464}{81} \frac{\widetilde{\alpha}^{2}}{\epsilon}+\frac{\widetilde{\alpha}^{3}}{\epsilon}\left[\frac{1}{\epsilon}\left(-\frac{118976}{2187}-\frac{10112}{729} f+40 \bar{Q}\right)+\frac{257344}{729}+\frac{16832}{2187} f-\frac{2288}{9} \bar{Q}\right]+\frac{\widetilde{\alpha}^{4}}{\epsilon}\left\{\frac { 1 } { \epsilon ^ { 2 } } \left[\frac{28293352}{19683}\right.\right. \\
& \left.+\quad \frac{201176}{2187} f-\frac{32008}{2187} f^{2}-\left(\frac{6160}{9}-\frac{140}{3} f\right) \bar{Q}\right]+\frac{1}{\epsilon}\left[-\frac{17496454}{2187}+\frac{3399452}{19683} f+\frac{83368}{6561} f^{2}+\left(\frac{296492}{81}\right.\right. \\
& \left.\left.-\quad \frac{752}{3} f\right) \bar{Q}\right]+\frac{1744567561}{177147}+\frac{9416120}{2187} \zeta_{3}-\left(\frac{59440775}{118098}-\frac{4708}{243} \zeta_{3}\right) f-\left(\frac{92}{81}-\frac{16}{27} \zeta_{3}\right) f^{2} \\
& \left.-\left[\frac{1095949}{648}+\frac{1210}{9} \zeta_{3}-\left(\frac{1934}{27}-\frac{80}{9} \zeta_{3}\right) f\right] \bar{Q}\right\} \text {, } \\
& Z_{58}=\frac{\widetilde{\alpha}^{2}}{\epsilon}\left(\frac{2192}{27}+9 f\right)-\frac{\widetilde{\alpha}^{3}}{\epsilon}\left[\frac{1}{\epsilon}\left(\frac{713164}{729}+\frac{5494}{243} f-8 f^{2}\right)-\frac{452714}{243}-\frac{97043}{1458} f+\frac{7}{3} f^{2}\right]+\frac{\widetilde{\alpha}^{4}}{\epsilon}\left[\frac { 1 } { \epsilon ^ { 2 } } \left(\frac{87867110}{6561}\right.\right. \\
& \left.-\frac{1639918}{2187} f-\frac{21299}{162} f^{2}+\frac{43}{6} f^{3}\right)-\frac{1}{\epsilon}\left(\frac{25933753}{729}-\frac{115452017}{52488} f-\frac{684959}{2916} f^{2}+\frac{28}{9} f^{3}\right) \\
& \left.+\frac{11439938489}{236196}-\frac{11082938}{729} \zeta_{3}-\left(\frac{269786863}{629856}+\frac{288731}{162} \zeta_{3}\right) f-\left(\frac{86423}{648}+\frac{635}{9} \zeta_{3}\right) f^{2}-\frac{37}{54} f^{3}\right], \\
& Z_{67}=-\frac{\widetilde{\alpha}^{2}}{\epsilon}\left(\frac{3008}{243}-\frac{80}{81} f-12 \bar{Q}\right)+\frac{\widetilde{\alpha}^{3}}{\epsilon}\left\{\frac{1}{\epsilon}\left[\frac{925216}{6561}-\frac{19720}{729} f+\frac{800}{729} f^{2}+\left(-\frac{404}{3}+\frac{32}{3} f\right) \bar{Q}\right]-\frac{389636}{2187}\right. \\
& \left.+\quad \frac{154280}{6561} f+\frac{448}{2187} f^{2}-\left(\frac{1468}{27}+\frac{28}{9} f\right) \bar{Q}\right\}+\frac{\widetilde{\alpha}^{4}}{\epsilon}\left\{\frac { 1 } { \epsilon ^ { 2 } } \left[-\frac{82789640}{59049}+\frac{8283332}{19683} f-\frac{234328}{6561} f^{2}+\frac{2080}{2187} f^{3}\right.\right. \\
& \left.+\left(\frac{37826}{27}-\frac{1885}{9} f+8 f^{2}\right) \bar{Q}\right]+\frac{1}{\epsilon}\left[\frac{20027615}{6561}-\frac{39955153}{59049} f+\frac{708617}{19683} f^{2}+\frac{1424}{6561} f^{3}-\left(\frac{17755}{243}\right.\right. \\
& \left.\left.-\quad \frac{8992}{81} f+\frac{28}{9} f^{2}\right) \bar{Q}\right]-\frac{2247378701}{531441}-\frac{766276}{6561} \zeta_{3}+\left(\frac{195301451}{354294}-\frac{21056}{2187} \zeta_{3}\right) f-\left(\frac{1251734}{59049}\right. \\
& \left.\left.+\frac{1540}{243} \zeta_{3}\right) f^{2}-\frac{1432}{6561} f^{3}+\left[\frac{7375087}{3888}-\frac{40664}{27} \zeta_{3}-\left(\frac{62269}{324}+\frac{142}{27} \zeta_{3}\right) f-\frac{74}{81} f^{2}\right] \bar{Q}\right\}, \\
& Z_{68}=-\frac{\widetilde{\alpha}^{2}}{\epsilon}\left(\frac{1906}{81}-\frac{55}{27} f\right)+\frac{\widetilde{\alpha}^{3}}{\epsilon}\left[\frac{1}{\epsilon}\left(\frac{873038}{2187}-\frac{9530}{243} f+\frac{695}{486} f^{2}\right)-\frac{726064}{729}-\frac{410713}{17496} f-\frac{6031}{2916} f^{2}\right] \\
& +\frac{\widetilde{\alpha}^{4}}{\epsilon}\left[\frac{1}{\epsilon^{2}}\left(-\frac{131785021}{19683}+\frac{448189}{486} f-\frac{380411}{8748} f^{2}+\frac{80}{81} f^{3}\right)+\frac{1}{\epsilon}\left(\frac{184494545}{8748}-\frac{940982911}{629856} f+\frac{3173417}{104976} f^{2}\right.\right. \\
& \left.-\frac{2821}{972} f^{3}\right)-\frac{97818607289}{5668704}-\frac{4146212}{2187} \zeta_{3}+\left(\frac{3023834561}{3779136}+\frac{309493}{1458} \zeta_{3}\right) f-\left(\frac{3278851}{78732}-\frac{175}{324} \zeta_{3}\right) f^{2} \\
& \left.-\frac{997}{4374} f^{3}\right] \text {. }
\end{aligned}
$$

The $n$-loop $1 / \epsilon^{n}$ poles vanish in the above renormalization constants, which is a consequence of the lack of one-loop contributions to $Z_{i 7}$ and $Z_{i 8}$.

The operators $X_{i}(5.2)$ differ by a multiplicative factor of $1 / \widetilde{\alpha}$ from the bare versions of $Q_{7}$ and $Q_{8}$. We have used $X_{i}$ in the actual renormalization constant calculation. Each $n$-loop term is then of order $\widetilde{\alpha}^{n}$, which makes the simple matrix relation between renormalization constants and anomalous dimensions applicable. Such a relation up to three-loops has been given in Appendix B of Ref. [12]. Extending that result to four loops in the same notation (but with 
$\left.\kappa^{0 n}=0\right)$ yields

$$
\begin{aligned}
\hat{\gamma} & =2 \hat{a}^{11} g^{2}+g^{4}\left[4 \hat{a}^{12}-2 \hat{a}^{01} \hat{a}^{11}-2 \hat{a}^{11} \hat{a}^{01}-4 \kappa^{11} \hat{a}^{01}\right]+g^{6}\left[6 \hat{a}^{13}-4 \hat{a}^{12} \hat{a}^{01}-2 \hat{a}^{01} \hat{a}^{12}-4 \hat{a}^{02} \hat{a}^{11}\right. \\
& \left.-2 \hat{a}^{11} \hat{a}^{02}+2 \hat{a}^{01} \hat{a}^{11} \hat{a}^{01}+2 \hat{a}^{11}\left(\hat{a}^{01}\right)^{2}+2\left(\hat{a}^{01}\right)^{2} \hat{a}^{11}+4 \kappa^{11}\left(\hat{a}^{01}\right)^{2}-8 \kappa^{11} \hat{a}^{02}-8 \kappa^{12} \hat{a}^{01}\right] \\
& +g^{8}\left[8 \hat{a}^{14}-6 \hat{a}^{13} \hat{a}^{01}-2 \hat{a}^{01} \hat{a}^{13}-6 \hat{a}^{03} \hat{a}^{11}-2 \hat{a}^{11} \hat{a}^{03}-4 \hat{a}^{12} \hat{a}^{02}-4 \hat{a}^{02} \hat{a}^{12}+4 \hat{a}^{02} \hat{a}^{01} \hat{a}^{11}\right. \\
& +4 \hat{a}^{02} \hat{a}^{11} \hat{a}^{01}+2 \hat{a}^{01} \hat{a}^{12} \hat{a}^{01}+2 \hat{a}^{11} \hat{a}^{02} \hat{a}^{01}+2 \hat{a}^{01} \hat{a}^{02} \hat{a}^{11}+2 \hat{a}^{11} \hat{a}^{01} \hat{a}^{02}+2 \hat{a}^{01} \hat{a}^{11} \hat{a}^{02}+4 \hat{a}^{12}\left(\hat{a}^{01}\right)^{2} \\
& +2\left(\hat{a}^{01}\right)^{2} \hat{a}^{12}-2 \hat{a}^{01} \hat{a}^{11}\left(\hat{a}^{01}\right)^{2}-2\left(\hat{a}^{01}\right)^{2} \hat{a}^{11} \hat{a}^{01}-2 \hat{a}^{11}\left(\hat{a}^{01}\right)^{3}-2\left(\hat{a}^{01}\right)^{3} \hat{a}^{11}-12 \hat{a}^{03} \kappa^{11}-12 \hat{a}^{01} \kappa^{13} \\
& \left.-16 \hat{a}^{02} \kappa^{12}+8 \hat{a}^{02} \hat{a}^{01} \kappa^{11}+4 \hat{a}^{01} \hat{a}^{02} \kappa^{11}+8\left(\hat{a}^{01}\right)^{2} \kappa^{12}-4\left(\hat{a}^{01}\right)^{3} \kappa^{11}\right]+\mathcal{O}\left(g^{10}\right) .
\end{aligned}
$$

After calculating the ADM $\hat{\gamma}$ from Eq. (5.5), we multiply the dipole operators by $\widetilde{\alpha}$, which brings us to the normalization of $Q_{7}$ and $Q_{8}$ as in Eq. (2.2). Next, we pass from $\hat{\gamma}$ to $\hat{\gamma}^{\text {eff }}$, which amounts to performing a simple linear transformation 2 that stems from Eq. (2.3).

The obtained complete expressions for $\hat{\gamma}^{(n) \text { eff }}(n=0,1,2)$ in the $b \rightarrow s \gamma$ case $\left(f=5, \bar{Q}=\frac{1}{3}\right.$, $Q_{u}=\frac{2}{3}$ ) are as follows:

$\hat{\gamma}^{(0) \mathrm{eff}}=\left[\begin{array}{cccccccc}-4 & \frac{8}{3} & 0 & -\frac{2}{9} & 0 & 0 & -\frac{208}{243} & \frac{173}{162} \\ 12 & 0 & 0 & \frac{4}{3} & 0 & 0 & \frac{416}{81} & \frac{70}{27} \\ 0 & 0 & 0 & -\frac{52}{3} & 0 & 2 & -\frac{176}{81} & \frac{14}{27} \\ 0 & 0 & -\frac{40}{9} & -\frac{100}{9} & \frac{4}{9} & \frac{5}{6} & -\frac{152}{243} & -\frac{587}{162} \\ 0 & 0 & 0 & -\frac{256}{3} & 0 & 20 & -\frac{6272}{81} & \frac{6596}{27} \\ 0 & 0 & -\frac{256}{9} & \frac{56}{9} & \frac{40}{9} & -\frac{2}{3} & \frac{4624}{243} & \frac{4772}{81} \\ 0 & 0 & 0 & 0 & 0 & 0 & \frac{32}{3} & 0 \\ 0 & 0 & 0 & 0 & 0 & 0 & -\frac{32}{9} & \frac{28}{3}\end{array}\right]$

\footnotetext{
${ }^{2}$ See Eq. (38) of Ref. [7].
} 


$$
\begin{aligned}
& \hat{\gamma}^{(2) \mathrm{eff}}=\left[\begin{array}{ccccccccc}
-\frac{12773}{18} & \frac{745}{9} & \frac{63187}{13122} & -\frac{981796}{6561} & -\frac{202663}{52488} & -\frac{24973}{69984} & \frac{150994745}{1062882} & -\frac{138745277}{354294} \\
\frac{1177}{2} & 306 & \frac{110477}{2187} & \frac{133529}{8748} & -\frac{42929}{8748} & \frac{354319}{11664} & \frac{138336202}{177147} & -\frac{83347037}{472392} \\
0 & 0 & -\frac{3572528}{2187} & -\frac{58158773}{8748} & \frac{552601}{4374} & \frac{6989171}{11664} & -\frac{58397866}{177147} & \frac{5093967523}{944784} \\
0 & 0 & -\frac{1651004}{6561} & -\frac{155405353}{52488} & \frac{1174159}{52488} & \frac{10278809}{34992} & -\frac{5108749081}{2125764} & -\frac{9826683847}{5668704} \\
0 & 0 & -\frac{147978032}{2187} & -\frac{168491372}{2187} & \frac{11213042}{2187} & \frac{17850329}{2916} & \frac{5824017302}{177147} & \frac{24198694001}{118098} \\
0 & 0 & \frac{136797922}{6561} & -\frac{72614473}{13122} & -\frac{9288181}{6561} & -\frac{16664027}{17496} & \frac{3603565835}{531441} & -\frac{122956397803}{1417176} \\
0 & 0 & 0 & 0 & 0 & 0 & \frac{179768}{81} & -\frac{23444}{81} & \frac{192137}{81}
\end{array}\right] \\
& +S_{3}\left[\begin{array}{cccccccc}
\frac{1472}{3} & -\frac{4288}{9} & -\frac{1360}{81} & -\frac{776}{81} & \frac{124}{81} & \frac{100}{27} & \frac{1272596}{6561} & -\frac{2373229}{4374} \\
-2144 & -224 & \frac{2720}{27} & -\frac{2768}{27} & -\frac{248}{27} & -\frac{110}{9} & -\frac{2713672}{2187} & -\frac{682658}{729} \\
0 & 0 & -\frac{608}{27} & \frac{61424}{27} & -\frac{496}{27} & -\frac{2821}{9} & \frac{3236560}{2187} & -\frac{1051828}{729} \\
0 & 0 & \frac{88720}{81} & \frac{54272}{81} & -\frac{9274}{81} & -\frac{3100}{27} & \frac{2007886}{6561} & \frac{4858889}{2187} \\
0 & 0 & \frac{87040}{27} & \frac{324416}{27} & -\frac{13984}{27} & -\frac{31420}{9} & \frac{112180720}{2187} & -\frac{119487244}{729} \\
0 & 0 & \frac{721408}{81} & -\frac{166432}{81} & -\frac{95032}{81} & -\frac{7552}{27} & \frac{15361912}{6561} & -\frac{71430598}{2187} \\
0 & 0 & 0 & 0 & 0 & 0 & -\frac{21056}{27} & 0 \\
0 & 0 & 0 & 0 & 0 & 0 & \frac{22976}{81} & -\frac{48304}{27}
\end{array}\right] .
\end{aligned}
$$

For arbitrary values of $f, \bar{Q}$ and $Q_{u}$, the blocks $B^{(n)}(n=0,1,2)$ read

$$
\begin{aligned}
& B^{(0)}=\left[\begin{array}{cc}
\frac{8}{243}-\frac{4}{3} Q_{u} & \frac{173}{162} \\
-\frac{16}{81}+8 Q_{u} & \frac{70}{27} \\
-\frac{176}{81} & \frac{14}{27} \\
\frac{88}{243}-\frac{16}{81} f & \frac{74}{81}-\frac{49}{54} f \\
-\frac{6272}{81} & \frac{1736}{27}+36 f \\
\frac{3136}{243}-\frac{160}{81} f+48 \bar{Q} & \frac{2372}{81}+\frac{160}{27} f
\end{array}\right] \\
& B^{(1)}=\left[\begin{array}{cc}
\frac{12614}{2187}-\frac{64}{2187} f-\frac{374}{27} Q_{u}+\frac{2}{27} f Q_{u} & \frac{65867}{5832}+\frac{431}{5832} f \\
-\frac{2332}{729}+\frac{128}{729} f+\frac{136}{9} Q_{u}-\frac{4}{9} f Q_{u} & \frac{10577}{486}-\frac{917}{972} f \\
\frac{97876}{729}-\frac{4352}{729} f-\frac{112}{3} \bar{Q} & \frac{42524}{243}-\frac{2398}{243} f \\
-\frac{70376}{2187}-\frac{15788}{2187} f+\frac{32}{729} f^{2}-\frac{140}{9} \bar{Q} & -\frac{159718}{729}-\frac{39719}{5832} f-\frac{253}{486} f^{2} \\
\frac{1764752}{729}-\frac{65408}{729} f-\frac{3136}{3} \bar{Q} & \frac{2281576}{243}+\frac{140954}{243} f-14 f^{2} \\
\frac{4193840}{2187}-\frac{324128}{2187} f+\frac{896}{729} f^{2}-\frac{1136}{9} \bar{Q}-\frac{56}{3} f \bar{Q} & -\frac{3031517}{729}-\frac{15431}{1458} f-\frac{6031}{486} f^{2}
\end{array}\right],
\end{aligned}
$$




$$
\begin{aligned}
& {\left[\frac{77506102}{531441}-\frac{875374}{177147} f+\frac{560}{19683} f^{2}-\frac{9731}{162} Q_{u}+\frac{11045}{729} f Q_{u}+\frac{316}{729} f^{2} Q_{u}+\frac{3695}{486} \bar{Q}\right.} \\
& -\frac{15463055}{177147}+\frac{242204}{59049} f-\frac{1120}{6561} f^{2}+\frac{55748}{27} Q_{u}-\frac{33970}{243} f Q_{u}-\frac{632}{243} f^{2} Q_{u}-\frac{3695}{81} \bar{Q} \\
& B^{(2)}=\mid \begin{array}{l}
\frac{102439553}{177147}-\frac{12273398}{59049} f+\frac{5824}{6561} f^{2}+\frac{26639}{81} \bar{Q}-\frac{8}{27} f \bar{Q} \\
-\frac{2493414077}{1062882}-\frac{9901031}{354294} f+\frac{243872}{59049} f^{2}-\frac{1184}{6561} f^{3}-\frac{49993}{972} \bar{Q}+\frac{305}{27} f \bar{Q}
\end{array} \\
& \frac{8808397748}{177147}-\frac{174839456}{59049} f+\frac{1600}{729} f^{2}-\frac{669694}{81} \bar{Q}+\frac{10672}{27} f \bar{Q} \\
& \frac{7684242746}{531441}-\frac{351775414}{177147} f-\frac{479776}{59049} f^{2}-\frac{11456}{6561} f^{3}+\frac{3950201}{243} \bar{Q}-\frac{130538}{81} f \bar{Q}-\frac{592}{81} f^{2} \bar{Q} \\
& -\frac{421272953}{1417176}-\frac{8210077}{472392} f-\frac{1955}{6561} f^{2} \\
& \frac{98548513}{472392}-\frac{5615165}{78732} f-\frac{2489}{2187} f^{2} \\
& \frac{3205172129}{472392}-\frac{108963529}{314928} f+\frac{58903}{4374} f^{2} \\
& -\frac{6678822461}{2834352}+\frac{127999025}{1889568} f+\frac{1699073}{157464} f^{2}+\frac{505}{4374} f^{3} \\
& \frac{29013624461}{118098}-\frac{64260772}{19683} f-\frac{230962}{243} f^{2}-\frac{148}{27} f^{3} \\
& \left.-\frac{72810260309}{708588}+\frac{2545824851}{472392} f-\frac{33778271}{78732} f^{2}-\frac{3988}{2187} f^{3}\right] \\
& +\zeta_{3}\left[\begin{array}{ll}
-\frac{112216}{6561}+\frac{728}{729} f+\frac{25508}{81} Q_{u}-\frac{64}{81} f Q_{u}-\frac{100}{27} \bar{Q} & -\frac{953042}{2187}-\frac{10381}{486} f \\
\frac{365696}{2187}-\frac{1168}{243} f-\frac{51232}{27} Q_{u}-\frac{1024}{27} f Q_{u}+\frac{200}{9} \bar{Q} & -\frac{607103}{729}-\frac{1679}{81} f \\
\frac{3508864}{2187}-\frac{1904}{243} f-\frac{1984}{9} \bar{Q}-\frac{64}{9} f \bar{Q} & -\frac{1597588}{729}+\frac{13028}{81} f-\frac{20}{9} f^{2} \\
-\frac{1922264}{6561}+\frac{308648}{2187} f-\frac{1280}{243} f^{2}+\frac{1010}{9} \bar{Q}-\frac{200}{27} f \bar{Q} & \frac{2312684}{2187}+\frac{128347}{729} f+\frac{920}{81} f^{2} \\
\frac{123543040}{2187}-\frac{207712}{243} f+\frac{128}{27} f^{2}-\frac{24880}{9} \bar{Q}-\frac{640}{9} f \bar{Q} & -\frac{69359224}{729}-\frac{885356}{81} f-\frac{5080}{9} f^{2} \\
\frac{7699264}{6561}+\frac{2854976}{2187} f-\frac{12320}{243} f^{2}-\frac{108584}{9} \bar{Q}-\frac{1136}{27} f \bar{Q} & -\frac{61384768}{2187}-\frac{685472}{729} f+\frac{350}{81} f^{2}
\end{array}\right]
\end{aligned}
$$

The $f$ - and charge-dependence of the blocks $C^{(n)}$ for $n=0,1,2$ can be found in Eqs. (9)(11) of Ref. [10]. The blocks $A^{(n)}$ are charge-independent, while their $f$-dependence is given in Eqs. (36)-(38) of Ref. [9].

\section{Numerical Analysis}

Let $\mathcal{B}_{\mathrm{NNLO}}$ denote the NNLO value of the $\bar{B} \rightarrow X_{s} \gamma$ branching ratio, and the superscript " $4 L \rightarrow 0$ " indicate that the four-loop matrix $B^{(2)}$ is set to zero in the calculation of a given quantity. Normalizing the difference between $\mathcal{B}_{\mathrm{NNLO}}$ and $\mathcal{B}_{\mathrm{NNLO}}^{4 \mathrm{~L} \rightarrow 0}$ to the LO result, one finds

$$
\frac{\mathcal{B}_{\mathrm{NNLO}}-\mathcal{B}_{\mathrm{NNLO}}^{4 \mathrm{~L} \rightarrow 0}}{\mathcal{B}_{\mathrm{LO}}}=\left(\frac{\alpha_{s}\left(\mu_{b}\right)}{\pi}\right)^{2} \frac{C_{7}^{(2) \mathrm{eff}}\left(\mu_{b}\right)-\left[C_{7}^{(2) \mathrm{eff}}\left(\mu_{b}\right)\right]^{4 \mathrm{~L} \rightarrow 0}}{8 C_{7}^{(0) \mathrm{eff}}\left(\mu_{b}\right)}
$$




\begin{tabular}{|l|rrrrrrrr|}
\hline$i$ & 1 & 2 & 3 & 4 & 5 & 6 & 7 & 8 \\
\hline$a_{i}$ & $\frac{14}{23}$ & $\frac{16}{23}$ & $\frac{6}{23}$ & $-\frac{12}{23}$ & 0.4086 & -0.4230 & -0.8994 & 0.1456 \\
$h_{i}^{(0)}$ & 2.2996 & -1.0880 & -0.4286 & -0.0714 & -0.6494 & -0.0380 & -0.0185 & -0.0057 \\
$h_{i}^{(1)}$ & 0.9075 & -0.7550 & 0.6971 & 0.0166 & -0.7345 & 0.1784 & -0.2608 & -0.0493 \\
$h_{i}^{(2)}$ & -13.9117 & 11.4063 & 3.4986 & -0.0619 & -2.4429 & 2.1210 & -0.7558 & 0.1464 \\
\hline
\end{tabular}

Table 1: The numbers $a_{i}$ and $h_{i}^{(n)}$ that occur in Eqs. (6.6) and (6.8).

where $\mu_{b}$ is the low-energy scale at which the RGEs evolution is terminated, while $C_{7}^{(n) \text { eff }}$ are defined by the perturbative expansion

$$
C_{7}^{\mathrm{eff}}(\mu)=\sum_{n \geq 0} \widetilde{\alpha}^{n}(\mu) C_{7}^{(n) \mathrm{eff}}(\mu) .
$$

To evaluate the r.h.s. of Eq. (6.1), we need to know the non-vanishing LO initial conditions for the Wilson coefficients at the electroweak scale $\mu_{0}$. They read [19]

$$
\begin{aligned}
& C_{2}^{(0)}\left(\mu_{0}\right)=1, \\
& C_{7}^{(0)}\left(\mu_{0}\right)=\frac{3 x^{3}-2 x^{2}}{4(x-1)^{4}} \ln x+\frac{-8 x^{3}-5 x^{2}+7 x}{24(x-1)^{3}}, \\
& C_{8}^{(0)}\left(\mu_{0}\right)=\frac{-3 x^{2}}{4(x-1)^{4}} \ln x+\frac{-x^{3}+5 x^{2}+2 x}{8(x-1)^{3}},
\end{aligned}
$$

where $x=m_{t}^{2}\left(\mu_{0}\right) / M_{W}^{2}$. Substituting them into the analytical solution to the RGEs (see, e.g., Section 3.3 of Ref. [18]), one obtains

$$
\frac{C_{7}^{(2) \mathrm{eff}}\left(\mu_{b}\right)-\left[C_{7}^{(2) \mathrm{eff}}\left(\mu_{b}\right)\right]^{4 \mathrm{~L} \rightarrow 0}}{8 C_{7}^{(0) \mathrm{eff}}\left(\mu_{b}\right)}=\frac{h_{1}^{(2)} \eta^{a_{1}+2}+h_{2}^{(2)} \eta^{a_{2}+2}+\sum_{i=3}^{8} h_{i}^{(2)} \eta^{a_{i}}}{\eta^{a_{2}} C_{7}^{(0)}\left(\mu_{0}\right)+\frac{8}{3}\left(\eta^{a_{1}}-\eta^{a_{2}}\right) C_{8}^{(0)}\left(\mu_{0}\right)+\sum_{i=1}^{8} h_{i}^{(0)} \eta^{a_{i}}},
$$

where $\eta=\alpha_{s}\left(\mu_{0}\right) / \alpha_{s}\left(\mu_{b}\right)$. For the numerical evaluation of $\eta$, we always use the four-loop RGE for $\alpha_{s}(\mu)$. The numbers $a_{i}$ and $h_{i}^{(n)}$ are given in Table 1. They are found from the diagonalization of $\hat{\gamma}^{(0) \text { eff }}$ (5.6) as well as the elements of $\hat{\gamma}^{(1) \text { eff }}$ (5.7) and $\hat{\gamma}^{(2) \text { eff }}$ (5.8). Although $\hat{\gamma}^{(0) \text { eff }}$ can be diagonalized analytically, the decimal approximations presented in Table 1 are much more convenient to use. Note that $\sum_{i=1}^{8} h_{i}^{(n)}=0$, which follows from the initial condition for $C_{7}$ at $\eta=1$ and from the off-diagonal position of $B^{(n)}$ in $\hat{\gamma}^{(n) \text { eff }}$.

The r.h.s. of Eq. (6.6) is shown in the left plot of Fig. 2 as a function of $\eta$ for $x=(162 / 80.4)^{2}$. The middle plot in the same figure presents this quantity as a function of $\mu_{b}$ for $\mu_{0}=160 \mathrm{GeV}$ and for the remaining input parameters as listed in Appendix A of Ref. [4]. The $\mu_{b}$-dependence of the complete correction (6.1) is shown for the same parameters in the right plot of Fig. 2,

It is interesting to compare the four-loop effect to the analogous three-loop one at the Nextto-Leading Order (NLO). Let the superscript " $3 L \rightarrow 0$ " indicate neglecting the three-loop matrix $B^{(1)}$. Then

$$
\frac{\mathcal{B}_{\mathrm{NLO}}-\mathcal{B}_{\mathrm{NLO}}^{3 \mathrm{~L} \rightarrow 0}}{\mathcal{B}_{\mathrm{LO}}}=\frac{\alpha_{s}\left(\mu_{b}\right)}{\pi} \frac{C_{7}^{(1) \mathrm{eff}}\left(\mu_{b}\right)-\left[C_{7}^{(1) \mathrm{eff}}\left(\mu_{b}\right)\right]^{3 \mathrm{~L} \rightarrow 0}}{2 C_{7}^{(0) \mathrm{eff}}\left(\mu_{b}\right)}
$$


and

$$
\frac{C_{7}^{(1) \mathrm{eff}}\left(\mu_{b}\right)-\left[C_{7}^{(1) \mathrm{eff}}\left(\mu_{b}\right)\right]^{3 \mathrm{~L} \rightarrow 0}}{2 C_{7}^{(0) \mathrm{eff}}\left(\mu_{b}\right)}=\frac{h_{1}^{(1)} \eta^{a_{1}+1}+h_{2}^{(1)} \eta^{a_{2}+1}+\sum_{i=3}^{8} h_{i}^{(1)} \eta^{a_{i}}}{\eta^{a_{2}} C_{7}^{(0)}\left(\mu_{0}\right)+\frac{8}{3}\left(\eta^{a_{1}}-\eta^{a_{2}}\right) C_{8}^{(0)}\left(\mu_{0}\right)+\sum_{i=1}^{8} h_{i}^{(0)} \eta^{a_{i}}}
$$

The ratios (6.7) and (6.8) are shown in Fig. 3 as functions of $\eta$ and $\mu_{b}$.
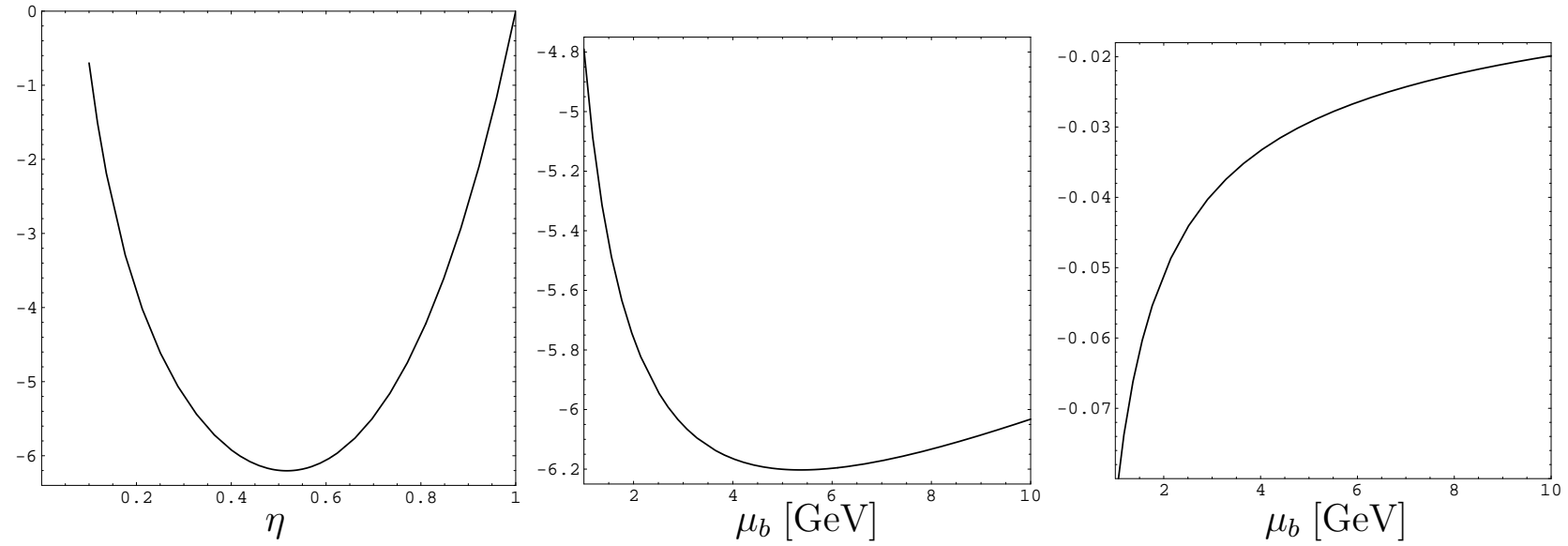

Figure 2: The r.h.s. of Eq. (6.6) as a function of $\eta$ (left plot) and $\mu_{b}$ (middle plot). The right plot shows the relative NNLO correction (6.1) to the branching ratio as a function of $\mu_{b}$.
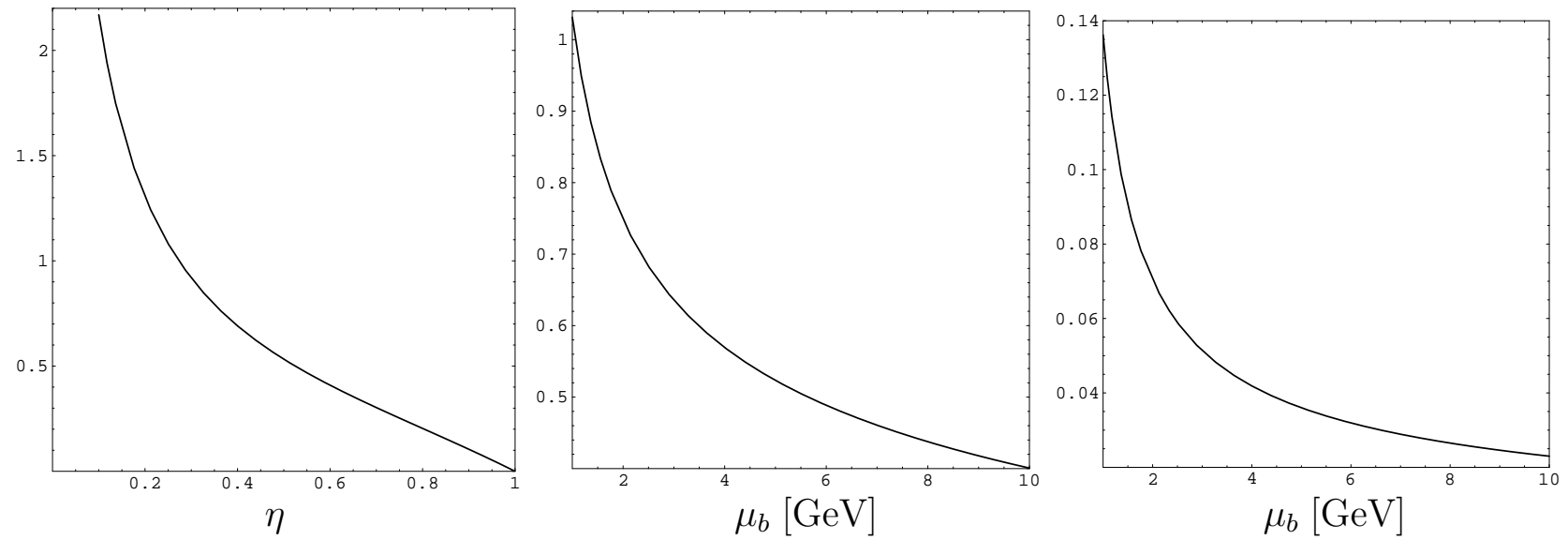

Figure 3: The r.h.s. of Eq. (6.8) as a function of $\eta$ (left plot) and $\mu_{b}$ (middle plot). The right plot shows the relative NLO correction (6.7) to the branching ratio as a function of $\mu_{b}$.

Comparing the right plots in Figs. 2 and 3, one can see that the two corrections are similar in magnitude. The suppression of the NNLO one by an additional factor of $\alpha_{s}\left(\mu_{b}\right) / \pi$ tends to get compensated by larger values of $h_{i}^{(2)}$ as compared to $h_{i}^{(1)}$. However, as the remaining plots show, the ratios (6.6) and (6.8) do not contradict the naive expectation of being "numbers of order unity". Thus, there seems to be no deep reason behind the observed relative smallness of 
the NLO effect and the relatively large value of the NNLO one. It should be remembered that the other NLO corrections exceed $\sim 30 \%$ while the other NNLO ones stay within $\sim 10 \%$ [3].

It is evident from Fig. 2 that the $\mu_{b}$-dependence of the NNLO correction (6.1) for $\mu_{b} \in$ $[1,10] \mathrm{GeV}$ originates almost entirely from the overall factor $\alpha_{s}^{2}\left(\mu_{b}\right)$. The $\pm 3 \%$ higher-order uncertainty in $\mathcal{B}_{\text {NNLO }}$ that was estimated in Refs. $[3,4]$ takes this $\mu_{b}$-dependence into account. The central value of $\mathcal{B}_{\mathrm{NNLO}}$ was calculated there for $\mu_{b}=2.5 \mathrm{GeV}$. At that scale, our present correction amounts to around $-4.4 \%$. It is worth noting that $\mathcal{B}_{\mathrm{NNLO}}^{4 \mathrm{~L} \rightarrow 0}$ calculated along the formulae of Ref. [4] turns out to be much less $\mu_{b}$-dependent than the more complete $\mathcal{B}_{\text {NNLO }}$.

The actual numerical calculation in Refs. [3,4] included contributions from $B_{i 7}^{(2)}$ but not from the very recently found $B_{i 8}^{(2)}$. This fact is practically irrelevant for the above discussion. The numerical effect of $B_{i 8}^{(2)}$ on $\mathcal{B}_{\text {NNLO }}$ is about 10 times smaller than the one of $B_{i 7}^{(2)}$, and has the same sign. The small contribution of $B_{i 8}^{(2)}$ will be taken into account together with other similar ones in a future upgrade of the phenomenological NNLO analysis.

\section{Summary}

We have evaluated the complete four-loop anomalous dimension matrix $B^{(2)}$ that is necessary for determining the effective FCNC couplings $\bar{q} q^{\prime} \gamma$ and $\bar{q} q^{\prime} g$ at the NNLO in QCD. The results are presented in a form that applies to any external flavour case, with an arbitrary number of non-decoupled quarks. Adding an essential contribution to the NNLO QCD analysis $\bar{B} \rightarrow X_{s} \gamma$ has been the main purpose of our calculation. The obtained $\mathcal{O}\left(\alpha_{s}^{2}\left(\mu_{b}\right)\right)$ correction to the branching ratio of this decay amounts to around $-2.9 \%$ for $\mu_{b}=5 \mathrm{GeV}$, and $-4.4 \%$ for $\mu_{b}=2.5 \mathrm{GeV}$.

\section{Acknowledgments}

The work of M.C. was supported by the Sofja Kovalevskaja Award of the Alexander von Humboldt Foundation sponsored by the German Federal Ministry of Education and Research. U.H. was supported by the Swiss National Foundation. M.M. acknowledges support by the Polish Committee for Scientific Research under the grant 2 P03B 078 26, and from the EU Contract MRTN-CT-2006-035482, FLAVIAnet. Our calculations made extensive use of the DESYZeuthen Grid Engine computer cluster, the Fermilab General-Purpose Computing Farms [20], as well as the Z-box computer at the University of Zürich [21].

\section{Appendix A}

This appendix contains solutions to the RGEs for all the Wilson coefficients that matter for $b \rightarrow s \gamma$ at the NNLO before including higher-order electroweak corrections. A generic solution to the RGEs (2.4) reads

$$
\vec{C}^{\mathrm{eff}}\left(\mu_{b}\right)=\hat{U}\left(\mu_{b}, \mu_{0}\right) \vec{C}^{\mathrm{eff}}\left(\mu_{0}\right) .
$$




\begin{tabular}{|c|c|c|c|}
\hline \multirow{2}{*}{$k$} & \multicolumn{3}{|c|}{$l$} \\
\cline { 2 - 4 } & $n=0$ & $n=1$ & $n=2$ \\
\hline 1,2 & 1,2 & 2 & none \\
$3,4,5,6$ & $1,2,4$ & 2 & none \\
7 & all & $1,2,4,7,8$ & $2,7,8$ \\
8 & $1,2,4,8$ & 2,8 & none \\
\hline
\end{tabular}

Table 2: Indices $k$ and $l$ of the relevant $U_{k l}^{(n)}$ for $n=0,1,2$.

The block-triangular structure of the matrix $\hat{U}$ corresponds to that of $\left(\hat{\gamma}^{\text {eff }}\right)^{T}$, taking into account that the blocks $A^{(n)}$ and $C^{(n)}$ in Eq. (2.6) have a block-triangular form, too. In particular, $U_{87}=0$, and $U_{k l}=0$ for $k \leq 2$ and $l>2$.

Using the perturbative expansions for $C_{i}^{\text {eff }}(\mu)(\underline{6.2})$ and

$$
\hat{U}\left(\mu_{b}, \mu_{0}\right)=\sum_{n \geq 0} \widetilde{\alpha}^{n}\left(\mu_{0}\right) \hat{U}^{(n)},
$$

one easily finds that

$$
\begin{aligned}
\vec{C}^{(0) \mathrm{eff}}\left(\mu_{b}\right) & =\hat{U}^{(0)} \vec{C}^{(0) \mathrm{eff}}\left(\mu_{0}\right) \\
\vec{C}^{(1) \mathrm{eff}}\left(\mu_{b}\right) & =\eta\left[\hat{U}^{(0)} \vec{C}^{(1) \mathrm{eff}}\left(\mu_{0}\right)+\hat{U}^{(1)} \vec{C}^{(0) \mathrm{eff}}\left(\mu_{0}\right)\right] \\
\vec{C}^{(2) \mathrm{eff}}\left(\mu_{b}\right) & =\eta^{2}\left[\hat{U}^{(0)} \vec{C}^{(2) \mathrm{eff}}\left(\mu_{0}\right)+\hat{U}^{(1)} \vec{C}^{(1) \mathrm{eff}}\left(\mu_{0}\right)+\hat{U}^{(2)} \vec{C}^{(0) \mathrm{eff}}\left(\mu_{0}\right)\right] .
\end{aligned}
$$

The matrices $\hat{U}^{(n)}$ are functions of $\eta$ only

$$
U_{k l}^{(n)}=\sum_{j=0}^{n} \sum_{i=1}^{8} m_{k l i}^{(n j)} \eta^{a_{i}-j} .
$$

The powers $a_{i}$ have been given in Table 1, Only a relatively small set of non-vanishing $U_{k l}^{(n)}$ is relevant for $b \rightarrow s \gamma$ at the NNLO, so long as

$$
\begin{aligned}
& C_{l}^{(0) \text { eff }}\left(\mu_{0}\right)=0, \quad \text { for } l=1,3,4,5,6, \\
& C_{l}^{(1) \text { eff }}\left(\mu_{0}\right)=0, \quad \text { for } l=3,5,6,
\end{aligned}
$$

which is the case in the SM. The corresponding values of the indices $k$ and $l$ for $n=0,1,2$ are collected in Table 2, The "magic numbers" $m_{k l i}^{(n j)}$ that occur in the expressions (A.6) for these $U_{k l}^{(n)}$ are given in Tables 35 .

As far as $C_{i}^{(n) \text { eff }}\left(\mu_{0}\right)$ are concerned, their SM values for $i=1, \ldots, 6$ can be found in Section 2 of Ref. [16]. The coefficients $C_{i}^{Q(n)}$ from that paper combine with a relative minus sign to our $C_{i}^{(n)}$, i.e. $C_{i}^{(n)}=C_{i}^{t(n)}-C_{i}^{c(n)}$. For $i=7,8$, one can find the corresponding $C_{i}^{Q(n)}$ in Section 6 of Ref. [17]. They combine to our $C_{i}^{(n)}$ according to the relation $C_{i}^{(n)}=C_{i}^{t(n+1)}-C_{i}^{c(n+1)}$. The upper index gets shifted because $Q_{7}$ and $Q_{8}$ in Ref. [17] were normalized as $X_{7}$ and $X_{8}$ in Eq. (5.2) here. Once $C_{i}^{(n)}\left(\mu_{0}\right)$ are evaluated, Eq. (2.3) should be applied to obtain $C_{i}^{(n) \text { eff }}\left(\mu_{0}\right)$. 


\section{References}

[1] S. Bertolini, F. Borzumati, A. Masiero and G. Ridolfi, Nucl. Phys. B 353 (1991) 591.

[2] P. L. Cho and M. Misiak, Phys. Rev. D 49 (1994) 5894 [hep-ph/9310332];

K. Fujikawa and A. Yamada, Phys. Rev. D 49 (1994) 5890.

[3] M. Misiak et al., hep-ph/0609232, to be published in the Phys. Rev. Lett.

[4] M. Misiak and M. Steinhauser, hep-ph/0609241, to be published in the Nucl. Phys. B.

[5] G. Buchalla, A. J. Buras and M. E. Lautenbacher, Rev. Mod. Phys. 68 (1996) 1125 [hep-ph/9512380].

[6] K.G. Chetyrkin, M. Misiak and M. Münz, Phys. Lett. B 400 (1997) 206, Phys. Lett. B 425 (1998) 414 (E) [hep-ph/9612313].

[7] A.J. Buras, M. Misiak, M. Münz and S. Pokorski, Nucl. Phys. B 424 (1994) 374 hep-ph/9311345].

[8] P. Gambino, M. Gorbahn and U. Haisch, Nucl. Phys. B 673 (2003) 238 hep-ph/0306079.

[9] M. Gorbahn and U. Haisch, Nucl. Phys. B 713 (2005) 291 hep-ph/0411071].

[10] M. Gorbahn, U. Haisch and M. Misiak, Phys. Rev. Lett. 95 (2005) 102004 hep-ph/0504194.

[11] M. Misiak and M. Münz, Phys. Lett. B 344 (1995) 308 hep-ph/9409454.

[12] K. G. Chetyrkin, M. Misiak and M. Münz, Nucl. Phys. B 518 (1998) 473 [hep-ph/9711266].

[13] T. van Ritbergen, J. A. M. Vermaseren and S. A. Larin, Phys. Lett. B 400 (1997) 379 [hep-ph/9701390].

[14] M. Czakon, Nucl. Phys. B 710 (2005) 485 [hep-ph/0411261].

[15] A.J. Buras and P.H. Weisz, Nucl. Phys. B 333 (1990) 66;

M. J. Dugan and B. Grinstein, Phys. Lett. B 256 (1990) 239.

[16] C. Bobeth, M. Misiak and J. Urban, Nucl. Phys. B 574 (2000) 291 hep-ph/9910220.

[17] M. Misiak and M. Steinhauser, Nucl. Phys. B 683 (2004) 277 [hep-ph/0401041].

[18] T. Huber, E. Lunghi, M. Misiak and D. Wyler, Nucl. Phys. B 740 (2006) 105 hep-ph/0512066.

[19] T. Inami and C. S. Lim, Prog. Theor. Phys. 65 (1981) 297, Prog. Theor. Phys. 65 (1981) $1772(\mathrm{E})$.

[20] M. Albert et al., FERMILAB-TM-2209.

[21] http://krone.physik.unizh.ch/ ^stadel/zbox/start 


\begin{tabular}{|c|c|c|c|c|c|c|c|c|}
\hline$i$ & 1 & 2 & 3 & 4 & 5 & 6 & 7 & 8 \\
\hline$m_{11 i}^{(00)}$ & 0 & 0 & 0.3333 & 0.6667 & 0 & 0 & 0 & 0 \\
\hline$m_{12 i}^{(00)}$ & 0 & 0 & 1 & -1 & 0 & 0 & 0 & 0 \\
\hline$m_{21 i}^{(00)}$ & 0 & 0 & 0.2222 & -0.2222 & 0 & 0 & 0 & 0 \\
\hline$m_{22 i}^{(00)}$ & 0 & 0 & 0.6667 & 0.3333 & 0 & 0 & 0 & 0 \\
\hline$m_{31 i}^{(00)}$ & 0 & 0 & 0.0106 & 0.0247 & -0.0129 & -0.0497 & 0.0092 & 0.0182 \\
\hline$m_{32 i}^{(00)}$ & 0 & 0 & 0.0317 & -0.0370 & -0.0659 & 0.0595 & -0.0218 & 0.0335 \\
\hline$m_{34 i}^{(00)}$ & 0 & 0 & 0 & 0 & -0.1933 & 0.1579 & 0.1428 & -0.1074 \\
\hline$m_{41 i}^{(00)}$ & 0 & 0 & 0.0159 & -0.0741 & 0.0046 & 0.0144 & 0.0562 & -0.0171 \\
\hline$m_{42 i}^{(00)}$ & 0 & 0 & 0.0476 & 0.1111 & 0.0237 & -0.0173 & -0.1336 & -0.0316 \\
\hline$m_{44 i}^{(00)}$ & 0 & 0 & 0 & 0 & 0.0695 & -0.0459 & 0.8752 & 0.1012 \\
\hline$m_{51 i}^{(00)}$ & 0 & 0 & -0.0026 & -0.0062 & 0.0018 & 0.0083 & -0.0004 & -0.0009 \\
\hline$m_{52 i}^{(00)}$ & 0 & 0 & -0.0079 & 0.0093 & 0.0094 & -0.0100 & 0.0010 & -0.0017 \\
\hline$m_{54 i}^{(00)}$ & 0 & 0 & 0 & 0 & 0.0274 & -0.0264 & -0.0064 & 0.0055 \\
\hline$m_{61 i}^{(00)}$ & 0 & 0 & -0.0040 & 0.0185 & 0.0021 & -0.0136 & -0.0043 & 0.0012 \\
\hline$m_{62 i}^{(00)}$ & 0 & 0 & -0.0119 & -0.0278 & 0.0108 & 0.0163 & 0.0103 & 0.0023 \\
\hline$m_{64 i}^{(00)}$ & 0 & 0 & 0 & 0 & 0.0317 & 0.0432 & -0.0675 & -0.0074 \\
\hline$m_{71 i}^{(00)}$ & 0.5784 & -0.3921 & -0.1429 & 0.0476 & -0.1275 & 0.0317 & 0.0078 & -0.0031 \\
\hline$m_{72 i}^{(00)}$ & 2.2996 & -1.0880 & -0.4286 & -0.0714 & -0.6494 & -0.0380 & -0.0185 & -0.0057 \\
\hline$m_{73 i}^{(00)}$ & 8.0780 & -5.2777 & 0 & 0 & -2.8536 & 0.1281 & 0.1495 & -0.2244 \\
\hline$m_{74 i}^{(00)}$ & 5.7064 & -3.8412 & 0 & 0 & -1.9043 & -0.1008 & 0.1216 & 0.0183 \\
\hline$m_{75 i}^{(00)}$ & 202.9010 & -149.4668 & 0 & 0 & -55.2813 & 2.6494 & 0.7191 & -1.5213 \\
\hline$m_{76 i}^{(00)}$ & 86.4618 & -59.6604 & 0 & 0 & -25.4430 & -1.2894 & 0.0228 & -0.0917 \\
\hline$m_{77 i}^{(00)}$ & 0 & 1 & 0 & 0 & 0 & 0 & 0 & 0 \\
\hline$m_{78 i}^{(00)}$ & 2.6667 & -2.6667 & 0 & 0 & 0 & 0 & 0 & 0 \\
\hline$m_{81 i}^{(00)}$ & 0.2169 & 0 & 0 & 0 & -0.1793 & -0.0730 & 0.0240 & 0.0113 \\
\hline$m_{82 i}^{(00)}$ & 0.8623 & 0 & 0 & 0 & -0.9135 & 0.0873 & -0.0571 & 0.0209 \\
\hline$m_{84 i}^{(00)}$ & 2.1399 & 0 & 0 & 0 & -2.6788 & 0.2318 & 0.3741 & -0.0670 \\
\hline$m_{88 i}^{(00)}$ & 1 & 0 & 0 & 0 & 0 & 0 & 0 & 0 \\
\hline
\end{tabular}

Table 3: "Magic numbers" for the relevant $U_{k l}^{(0)}$. 


\begin{tabular}{|c|c|c|c|c|c|c|c|c|}
\hline$i$ & 1 & 2 & 3 & 4 & 5 & 6 & 7 & 8 \\
\hline$m_{12 i}^{(10)}$ & 0 & 0 & -2.9606 & -4.0951 & 0 & 0 & 0 & 0 \\
\hline$m_{12 i}^{(11)}$ & 0 & 0 & 5.9606 & 1.0951 & 0 & 0 & 0 & 0 \\
\hline$m_{22 i}^{(10)}$ & 0 & 0 & -1.9737 & 1.3650 & 0 & 0 & 0 & 0 \\
\hline$m_{22 i}^{(11)}$ & 0 & 0 & 1.9737 & -1.3650 & 0 & 0 & 0 & 0 \\
\hline$m_{32 i}^{(10)}$ & 0 & 0 & -0.0940 & -0.1517 & -0.2327 & 0.2288 & 0.1455 & -0.4760 \\
\hline$m_{32 i}^{(11)}$ & 0 & 0 & -0.5409 & 1.6332 & 1.6406 & -1.6702 & -0.2576 & -0.2250 \\
\hline$m_{42 i}^{(10)}$ & 0 & 0 & -0.1410 & 0.4550 & 0.0836 & -0.0664 & 0.8919 & 0.4485 \\
\hline$m_{42 i}^{(11)}$ & 0 & 0 & 2.2203 & 2.0265 & -4.1830 & -0.7135 & -1.8215 & 0.7996 \\
\hline$m_{52 i}^{(10)}$ & 0 & 0 & 0.0235 & 0.0379 & 0.0330 & -0.0383 & -0.0066 & 0.0242 \\
\hline$m_{52 i}^{(11)}$ & 0 & 0 & 0.0400 & -0.1861 & -0.1669 & 0.1887 & 0.0201 & 0.0304 \\
\hline$m_{62 i}^{(10)}$ & 0 & 0 & 0.0352 & -0.1138 & 0.0382 & 0.0625 & -0.0688 & -0.0327 \\
\hline$m_{62 i}^{(11)}$ & 0 & 0 & -0.2614 & -0.1918 & 0.4197 & 0.0295 & 0.1474 & -0.0640 \\
\hline$m_{71 i}^{(10)}$ & 0.0021 & -1.4498 & 0.8515 & 0.0521 & 0.6707 & 0.1220 & -0.0578 & 0.0355 \\
\hline$m_{71 i}^{(11)}$ & -4.3519 & 3.0646 & 1.5169 & -0.5013 & 0.3934 & -0.6245 & 0.2268 & 0.0496 \\
\hline$m_{72 i}^{(10)}$ & 9.9372 & -7.4878 & 1.2688 & -0.2925 & -2.2923 & -0.1461 & 0.1239 & 0.0812 \\
\hline$m_{72 i}^{(11)}$ & -17.3023 & 8.5027 & 4.5508 & 0.7519 & 2.0040 & 0.7476 & -0.5385 & 0.0914 \\
\hline$m_{74 i}^{(10)}$ & -8.6840 & 8.5586 & 0 & 0 & 0.7579 & 0.4446 & 0.3093 & 0.4318 \\
\hline$m_{74 i}^{(11)}$ & -42.9356 & 30.0198 & 0 & 0 & 5.8768 & 1.9845 & 3.5291 & -0.2929 \\
\hline$m_{77 i}^{(10)}$ & 0 & 7.8152 & 0 & 0 & 0 & 0 & 0 & 0 \\
\hline$m_{77 i}^{(11)}$ & 0 & -7.8152 & 0 & 0 & 0 & 0 & 0 & 0 \\
\hline$m_{78 i}^{(10)}$ & 17.9842 & -18.7604 & 0 & 0 & 0 & 0 & 0 & 0 \\
\hline$m_{78 i}^{(11)}$ & -20.0642 & 20.8404 & 0 & 0 & 0 & 0 & 0 & 0 \\
\hline$m_{82 i}^{(10)}$ & 3.7264 & 0 & 0 & 0 & -3.2247 & 0.3359 & 0.3812 & -0.2968 \\
\hline$m_{82 i}^{(11)}$ & -5.8157 & 0 & 1.4062 & -3.9895 & 3.2850 & 3.6851 & -0.1424 & 0.6492 \\
\hline$m_{88 i}^{(10)}$ & 6.7441 & 0 & 0 & 0 & 0 & 0 & 0 & 0 \\
\hline$m_{88 i}^{(11)}$ & -6.7441 & 0 & 0 & 0 & 0 & 0 & 0 & 0 \\
\hline
\end{tabular}

Table 4: "Magic numbers" for the relevant $U_{k l}^{(1)}$. 


\begin{tabular}{|c|rrrrrrrr|}
\hline$i$ & 1 & 2 & 3 & 4 & 5 & 6 & 7 & 8 \\
\hline$m_{72 i}^{(20)}$ & -212.4136 & 167.6577 & 5.7465 & -3.7262 & 28.8574 & -2.1262 & 2.2903 & 0.1462 \\
$m_{72 i}^{(21)}$ & -74.7681 & 58.5182 & -13.4731 & 3.0791 & 7.0744 & 2.8757 & 3.5962 & -1.2982 \\
$m_{72 i}^{(22)}$ & 31.4443 & -18.1165 & 23.2117 & 13.2771 & -19.8699 & 4.0279 & -8.6259 & 2.6149 \\
$m_{77 i}^{(20)}$ & 0 & 44.4252 & 0 & 0 & 0 & 0 & 0 & 0 \\
$m_{77 i}^{(21)}$ & 0 & -61.0768 & 0 & 0 & 0 & 0 & 0 & 0 \\
$m_{77 i}^{(22)}$ & 0 & 16.6516 & 0 & 0 & 0 & 0 & 0 & 0 \\
$m_{78 i}^{(20)}$ & 15.4051 & -18.7662 & 0 & 0 & 0 & 0 & 0 & 0 \\
$m_{78 i}^{(21)}$ & -135.3141 & 146.6159 & 0 & 0 & 0 & 0 & 0 & 0 \\
$m_{78 i}^{(22)}$ & 36.4636 & -44.4043 & 0 & 0 & 0 & 0 & 0 & 0 \\
\hline
\end{tabular}

Table 5: "Magic numbers" for the relevant $U_{k l}^{(2)}$. 